\title{
The Essex-Lopresti injury: More than just a pain in the wrist
}

\author{
Shirley Hutchinson BSc Meds ${ }^{7}$, Kenneth J Faber MD MPHE FRCSC $1,2,4,6,7$, \\ Bing Siang Gan MD PhD FRCSC FACS $1,2,3,4,5,6,7$
}

\begin{abstract}
S Hutchinson, KJ Faber, BS Gan. The Essex-Lopresti injury: More than just a pain in the wrist. Can J Plast Surg 2006;14(4):215-218.
\end{abstract}

As the scope of plastic surgical practice expands to include disorders of the carpus and wrist, it has become increasingly important for plastic surgeons to understand pathoanatomy that has not traditionally been considered an integral component of training. The EssexLopresti injury consists of a radial head fracture with associated injury to the forearm interosseus membrane and longitudinal instability of the distal radioulnar joint. Early recognition of this disorder usually results in a predictable and satisfactory outcome. However, when this disorder is unrecognized, late reconstruction is challenging and unpredictable, and treatment may be misdirected to the wrist alone if the forearm and elbow are not considered as a component of this injury. The present report describes the importance of examining the elbow in all cases of wrist pain. As well, the literature is reviewed regarding the differences in treatment of acute and chronic EssexLopresti injuries. As plastic surgeons become more involved in the treatment of wrist injuries, the conscientious practitioner should be aware of more complicated pathology that may present as a seemingly straightforward wrist problem.

Key Words: DRUJ dislocation; Essex-Lopresti lesion; Forearm interosseus membrane; Radial head fracture; Wrist injury

$\mathrm{I}$

$\mathrm{n}$ North America, the independent specialty of hand surgery is a relatively new discipline. In Canada, surgery of the hand has traditionally been carried out by plastic surgeons, with a relatively sharp anatomic boundary just distal to the carpus. In contrast, hand surgery in the United States has a stronger basis in orthopedic surgery and, in addition to treatment of distal hand disorders, has always carried a stronger emphasis on problems involving the carpus and wrist. Because of several trends in the United States, including a new hand surgery certification requirement (the Certificate of Added Qualifications in Hand Surgery), and the maturation of scientific journals in the field of hand surgery, practice patterns in hand surgery in Canada are starting to mimic those in the United States and, as a result, the traditional treatment territory of the Canadian plastic surgeon is also expanding to include the carpus and wrist. Many Canadian plastic surgery training programs have recognized this and now include faculty who have received Certificate of Added Qualifications in Hand Surgery-eligible hand surgery training. Thus, a trend has been established in which plastic surgeons are seeing more patients with wrist pain and wrist trauma. Unfortunately, plastic surgeons may lack the necessary clinical skills required for the assessment of elbow

\section{Le syndrome d'Essex-Lopresti : plus qu'un simple mal de poignet}

À mesure que le champ de pratique de la chirurgie plastique s'élargit et englobe les lésions du carpe et du poignet, il importe de plus en plus que les chirurgiens plasticiens comprennent bien l'anatomopathologie de troubles qui, autrefois, ne faisaient pas partie de la formation de base. La fracture-luxation d'Essex-Lopresti consiste en une fracture de la tête radiale, associée à une lésion de la membrane interosseuse de l'avant-bras et à une instabilité longitudinale de l'articulation radio-cubitale distale. La reconnaissance et le traitement précoces de la lésion donnent généralement des résultats prévisibles et satisfaisants. Toutefois, lorsque la blessure passe inaperçue, la reconstruction tardive est difficile à réaliser et les résultats sont imprévisibles, et le traitement peut être mal orienté si l'avant-bras et le coude ne sont pas considérés dans l'ensemble du tableau. Le présent rapport fait état de l'importance d'examiner le coude dans tous les cas de douleur au poignet. À cela s'ajoute un examen de la documentation sur les différences de traitement entre les syndromes Essex-Lopresti aigu et chronique. Comme les chirurgiens plasticiens interviennent de plus en plus souvent dans le traitement des blessures au poignet, le praticien consciencieux devrait ne pas ignorer que des lésions plus complexes qu'elles ne le paraissent au départ peuvent se cacher derrière un simple mal de poignet. injuries that can occur in association with wrist trauma, because orthopedic specialists traditionally treat these injuries. As a result, plastic surgeons may fail to recognize that some wrist patients may suffer from complex forearm and elbow disorders that may extend the traditional 'comfortable' territory of the plastic surgeon into anatomical regions thus far foreign to plastic surgery. The present report describes one such patient and reviews the pathoanatomy, physiology and treatment of an often underdiagnosed disorder of the forearm.

\section{CASE PRESENTATION}

A 55-year-old, right hand-dominant man requested a second opinion consult for complaints of pain and disability in the right elbow and wrist. The patient had been seen elsewhere and was given a diagnosis of ulnocarpal impingement syndrome and advised to undergo an ulnar shortening osteotomy. On history, the patient had noticed a gradual decrease in range of motion and increasing discomfort in the elbow and wrist over the past three years. The pain was greater in the wrist than in the elbow and worsened during forceful grip.

The physical examination demonstrated a full range of motion in the shoulder. The elbow had normal flexion,

\footnotetext{
${ }^{1}$ The Hand and Upper Limb Centre, St Joseph's Health Centre; ${ }^{2}$ Department of Surgery; ${ }^{3}$ Divisions of Plastic and ${ }^{4}$ Orthopaedic Surgery;

${ }^{5}$ Departments of Physiology and Pharmacology; and ${ }^{6}$ Medical Biophysics; ${ }^{7}$ The University of Western Ontario, London, Ontario

Correspondence: Dr Bing Siang Gan, The Hand and Upper Limb Centre, 268 Grosvenor Street, London, Ontario N6A 4L6.

Telephone 519-646-6097, fax 519-646-6049, e-mail bsgan@rogers.com
} 


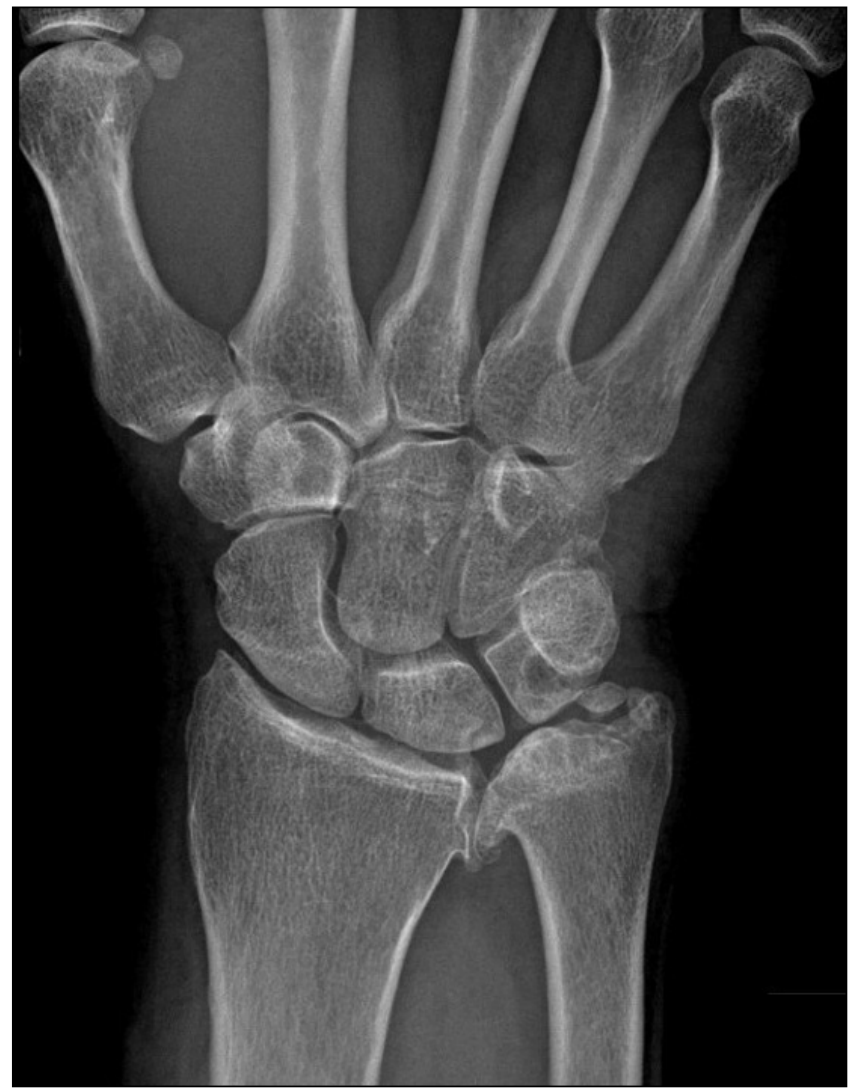

Figure 1) Posteroanterior view of affected right wrist showing marked ulnar-positive variance with degenerative arthritis of the distal radioulnar joint and the ulnocarpal joint

extension and pronation, but supination was limited to $50^{\circ}$. Palpation of the ulnar styloid and the dorso-ulnar aspect of the wrist in the region of the triangular fibrocartilage complex (TFCC) elicited tenderness. Pain was also reported during grip strength testing and was greater with the forearm in pronation than in supination.

His past medical history was significant for a remote fracture of his right elbow and a dislocation of his right wrist approximately 35 years previously in a motor vehicle accident. At that time, the patient had undergone surgical treatment of the elbow, which was described as reconstruction of the radial head. Despite this, pain persisted and three months later a radial head excision was performed. Over the ensuing 35 years, progressive pain in the elbow and wrist was treated with periodic steroid injections that provided temporary symptom relief. Over the past three years, there was progressive deterioration of the pain.

X-ray films of the right wrist (Figure 1) showed advanced degenerative arthritis in the distal radioulnar joint (DRUJ) and the ulnar head. There was a marked ulnar positive variance of $6 \mathrm{~mm}$. The ulnar variance of the unaffected left wrist (Figure 2) was $-3 \mathrm{~mm}$ and there was no degenerative arthritis of the DRUJ.

Plain radiographs of the right elbow (Figure 3) showed advanced degenerative arthritis in both the ulnohumeral and radiocapitellar joints. There were osteophytes on the coronoid and the olecranon, as well as several loose bodies in the elbow. There was an obviously fragmented radial head with subtotal resection of the proximal radius. These radiographic findings supported the diagnosis of an Essex-Lopresti injury.

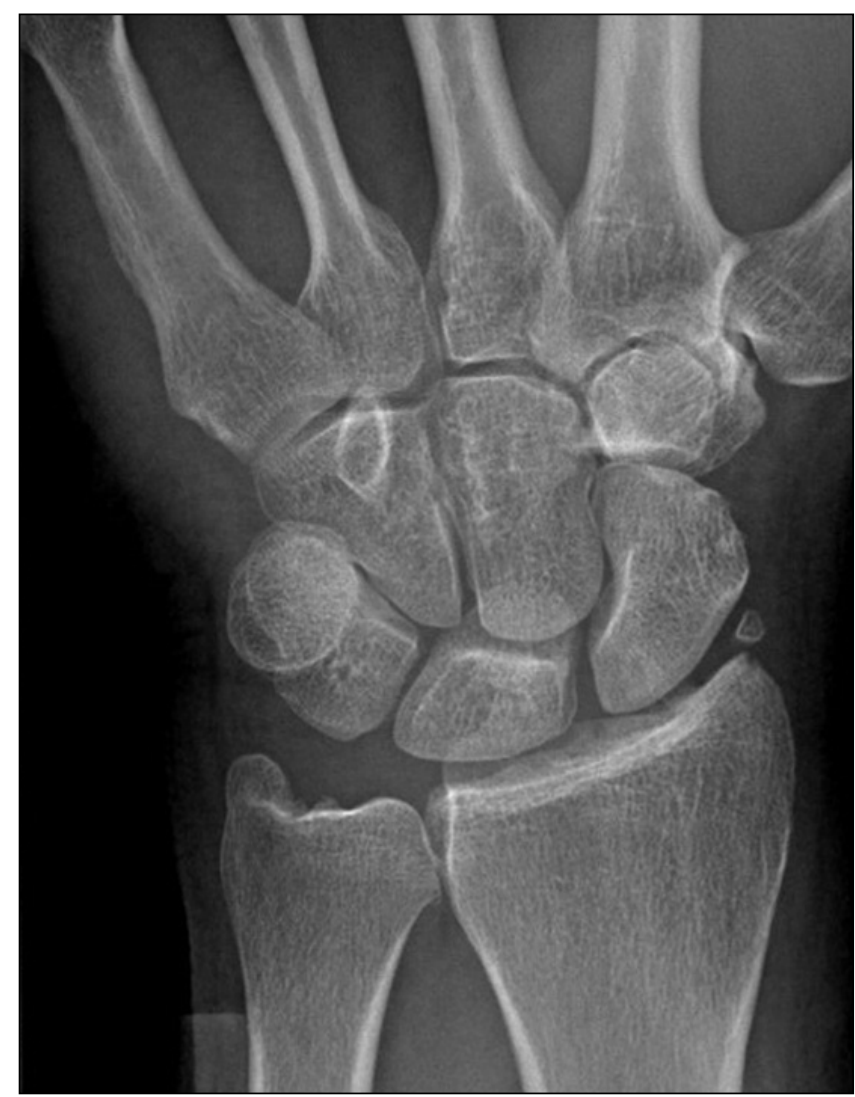

Figure 2) Posteroanterior view of contralateral left wrist showing normal ulnar variance and absence of degenerative arthritis

\section{DISCUSSION}

The Essex-Lopresti injury involves the combination of a fracture of the radial head, rupture of the forearm interosseous ligament (IOL) (the central band of the interosseous membrane) and DRUJ dislocation/subluxation. The result is longitudinal radioulnar dissociation and instability followed by proximal migration of the radius with respect to the ulna (1-3). The pattern of injury is very similar to the Maisonneuve fracture of the lower limb, which is characterized by a proximal fibular fracture, rupture of the interosseus membrane between the tibia and fibula, and distal tibiofibular dissociation (4).

The mechanism of injury for the Essex-Lopresti lesion requires a longitudinal force on the outstretched hand with the elbow in extension. Energy is transmitted proximally and the radial head is fractured after impacting on the capitellum. Forced forearm rotation disrupts the DRUJ and tears the interosseous membrane. Longitudinal stability of the forearm is disrupted and the radius can migrate proximally if the fracture fragments are significantly displaced, or if the radial head is surgically excised $(2,3)$.

The TFCC is the primary stabilizer of the DRUJ. The IOL also helps to stabilize the DRUJ. After the radial head is excised, the IOL and TFCC together maintain forearm stability and normally prevent proximal migration of the radius greater than $2 \mathrm{~mm}$. However, in the Essex-Lopresti injury, because of failure of both of these structures, proximal migration of the radius may occur (5). This proximal migration can occur at the time of injury or gradual migration of the radius can be seen months or years after radial head excision (3). 


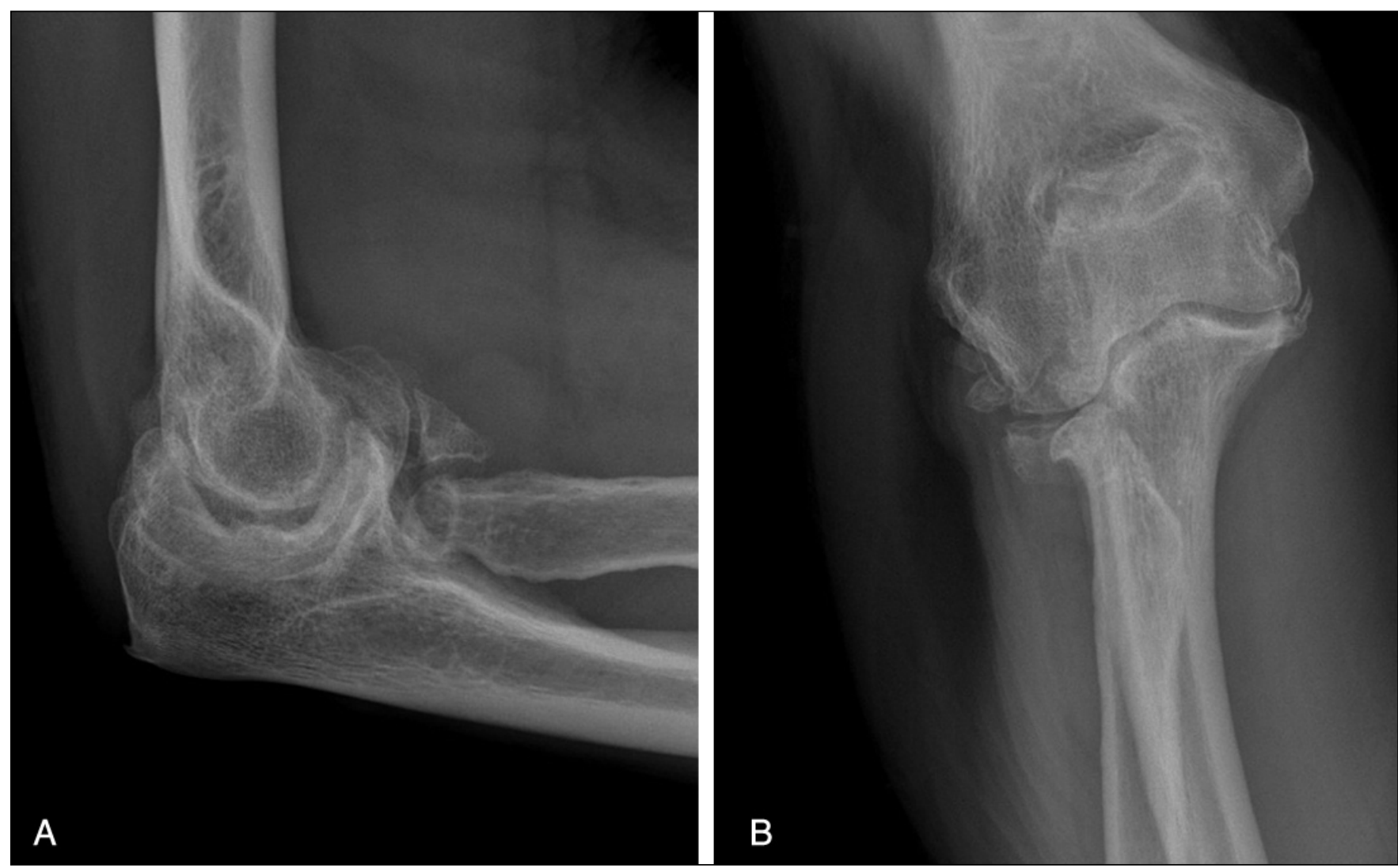

Figure 3) Lateral (A) and posteroanterior (B) views of affected right elbow showing degenerative changes in radiocapitellar and ulnohumeral joints with proximal radial fragmentation, partial removal of the radial head and proximal migration of the radius

In the acute setting, most attention is directed to the treatment of the radial head fracture, and the distal radioulnar dislocation/subluxation may be overlooked $(1,2,6)$. The wrist needs to be examined for instability and the forearm needs to be examined for pain over the IOL to avoid overlooking an underlying Essex-Lopresti injury. Patients with an acute EssexLopresti injury will present with symptoms at the DRUJ including tenderness, edema and instability. The dorsal surface of the distal ulna may also be painful (2). Following tearing of the interosseous membrane, proximal migration of the radius can result in ulnar positive variance of the wrist. A wrist radiograph and comparison views of the unaffected wrist $(2,3)$ may reveal the forearm instability pattern. Successful identification and treatment of the acute injury can help prevent patients from developing long-term sequelae.

The Essex-Lopresti injury is often undertreated in the acute stage. It is accepted that the best treatment outcomes occur when radial length is restored and the DRUJ is stabilized acutely. However, many of these patients will still have residual limitation in forearm rotation and grip strength. If treatment is delayed for more than six weeks, outcomes are generally poor (3). Delayed repair of an Essex-Lopresti fracture may result in irreducible proximal migration of the radius of as much as $20 \mathrm{~mm}(3,5)$ and other complications such as painful ulnar-sided wrist degeneration from ulnocarpal impingement $(3,7)$. Functional impairments such as decreased wrist extension, limited forearm rotation $(7,8)$ and chronic elbow pain $(1,5)$ can also develop.

Restoration of skeletal integrity of the radial head is the recommended treatment for acute Essex-Lopresti fractures
$(3,5)$. Displaced radial head fractures with little or no comminution can be repaired by open reduction and internal fixation (ORIF). This will restore normal anatomy and maintain normal radial length $(2,3,5)$. However, if the radial head is not reconstructable, the patient requires removal of the fragments and replacement with a prosthesis $(3,5)$. Silastic prostheses have not been shown to restore longitudinal stability to the forearm and are no longer advocated for use in the elbow if an Essex-Lopresti injury is suspected. In addition, silastic implants are prone to early mechanical failure, giving rise to deformation, dislocation, fragmentation and silicone synovitis $(3,5)$. Newer metallic prostheses are capable of preventing proximal migration of the radius. The modularity of metallic implants allows for precise implantation and anatomical reconstruction without performing excessive dissection that can further destabilize the elbow. Midterm survivorship of metallic implants are favourable, and complications are infrequent and include implant dislocation and metal wear on the articular cartilage, resulting in possible degenerative arthritis of the radiocapitellar joint $(2,5,9)$. Radial heads have also been replaced with frozen allografts but long-term results are not known (5).

The current thought on the treatment of Essex-Lopresti fractures is that, in addition to preventing proximal migration of the radius and DRUJ stabilization, restoration of load sharing between the radius and the ulna should be achieved $(3,5)$. Thus, ORIF of the radial head or radial head replacement to restore radial length should be followed by repair of the TFCC, and the DRUJ should be pinned or splinted in supination to reduce the joint and stabilize the forearm. This allows the ligaments to heal and prevents loss of supination $(2,3)$. When a 
radial head prosthesis is required, it may be necessary to reduce the radioulnar length and pin the DRUJ for four weeks (5).

The central one-third of the interosseous membrane transfers load from the distal radius to the proximal ulna. The radius is the chief load-bearing bone at the wrist but the IOL redistributes load so that it is equally shared between the radial head and the ulna at the elbow (5). Unfortunately, in contrast to the TFCC, the IOL has a limited ability to heal, with or without forearm immobilization, and IOL reconstruction is the treatment of choice (5). It is important to reconstruct the IOL without limiting forearm rotation (5). To prevent restriction of forearm rotation, grafts to the interosseous membrane should be tensioned in supination because in this position the strain is greater on the membrane than in pronation $(1,8)$. Reconstructing the IOL will restore load transfer between the radius and the ulna so that the radial head implant is unloaded. It is radiocapitellar contact at the elbow that impairs function at the elbow and results in arthritis (5). Tejwani et al (8) demonstrated that replacing the radial head with a metal implant and reconstructing the IOL greatly reduced mean distal ulnar force and may help reduce distal ulnar impaction.

Several materials have been investigated for IOL reconstruction. These include bone-patellar tendon-bone graft, nylon rope reconstruction, flexor carpi radialis (FCR) graft and Achilles tendon graft (10). Also, the use of the palmaris longus tendon (8) and hamstring tendon have been investigated (5). Stabile et al (11) found that the intact IOL was much stiffer than the Achilles tendon, the FCR and the bone-patellar tendonbone graft. Despite their conclusion that all grafts are structurally inferior to the IOL, they were also able to achieve full restoration of forearm load transfer by reconstructing the IOL with a double-stranded FCR graft (10).

When treating chronic Essex-Lopresti injuries, it is important to assess whether the proximal migration of the radius is fixed or reducible. If it is reducible, it is often sufficient to restore radioulnar length relations and obtain ulnar neutral variance, repair or replace the radial head, and pin the radius to the ulna at the DRUJ in supination, so that the TFCC and DRUJ capsule can heal (5).

If treatment has been delayed and the ulnar variance is not reducible, ulnar shortening osteotomy combined with radial head replacement to prevent further migration, or the use of an Ilizarov-type fixator for distraction lengthening of

\section{REFERENCES}

1. Gabriel MT, Pfaeffle HJ, Stabile KJ, Tomaino MM, Fischer KJ. Passive strain distribution in the interosseous ligament of the forearm: Implications for injury reconstruction. J Hand Surg [Am] 2004:29:293-8.

2. Morgan WJ, Breen TF. Complex fractures of the forearm. Hand Clin 1994;10:375-90.

3. Edwards GS Jr, Jupiter JB. Radial head fractures with acute distal radioulnar dislocation. Essex-Lopresti revisited. Clin Orthop Relat Res 1988;234:61-9.

4. Sproule JA, Khalid M, O'Sullivan M, McCabe JP. Outcome after surgery for Maisonneuve fracture of the fibula. Injury 2004;35:791-8.

5. Stabile KJ, Pfaeffle HJ, Tomaino MM. The Essex-Lopresti fracturedislocation factors in early management and salvage alternatives. Hand Clin 2002;18:195-204.

6. Malik AK, Pettit P, Compson J. Distal radioulnar joint dislocation in association with elbow injuries. Injury 2005;36:324-9.

7. Capuano L, Craig N, Ashcroft GP, Maffulli N. Distraction lengthening of the radius for radial longitudinal instability after the radius are needed to restore neutral ulnar variance (5). This improves wrist motion, forearm rotation and grip strength (3). Capuano et al (7) used Ilizarov external fixation to restore neutral ulnar variance at the wrist. A size-matched frozen radial head allograft was implanted and secured to the proximal radius with internal fixation. Their patient was effectively relieved of his wrist and elbow pain, however, the experience with frozen allograft has generally been poor (5). Szabo et al (12) also moved the radius distally using an Ilizarov technique and then replaced the radial head with a frozen allograft. Their five patients had relief of wrist and elbow pain as well as improved forearm rotation and wrist motion.

Finally, as a last resort, chronic Essex-Lopresti injuries can be salvaged by forming a one-bone forearm in a neutral or slightly pronated position but this solution abolishes forearm rotation, greatly reducing functionality (5). A one-bone forearm reconstruction also has a high rate of nonunion.

\section{CONCLUSION}

The critical step to successful treatment of an Essex-Lopresti lesion involves correct diagnosis of the injury. As the traditional treatment territory of the plastic surgeon extends more proximally and may include the forearm and wrist, surgeons must be careful to not overlook wrist injuries in all patients with elbow fractures and, conversely, rule out possible associated elbow injuries in their patients with wrist fractures and dislocations. Acute Essex-Lopresti injuries should be treated with radial head repair or replacement, ORIF of the DRUJ, and possibly TFCC repair. The IOL may be reconstructed using various materials, of which FCR tendon appears the most successful. The injury should ideally be treated acutely to avoid painful ulnar-sided wrist degeneration and chronic elbow pain. A chronic injury that was improperly diagnosed or treated may require radial head prosthetic replacement to stabilize the radius, in addition to either ulnar shortening osteotomy or the use of an Ilizarov-type lengthening device to restore neutral ulnar variance. Although reported to be successful in reducing wrist and elbow pain, these types of treatment are cumbersome, carry high complication rates and may eventually not achieve proper forearm function. Constructing a one-bone forearm is considered a last resort because it greatly decreases functionality.

distal radio-ulnar subluxation and excision of the radial head: A case report. Scand J Plast Reconstr Surg Hand Surg 2001;35:331-5.

8. Tejwani SG, Markolf KL, Benhaim P. Graft reconstruction of the interosseous membrane in conjunction with metallic radial head replacement: A cadaveric study. J Hand Surg [Am] 2005;30:335-42.

9. King GJ. Management of comminuted radial head fractures with replacement arthroplasty. Hand Clin 2004;20:429-41, vi.

10. Pfaeffle HJ, Stabile KJ, Li ZM, Tomaino MM. Reconstruction of the interosseous ligament restores normal forearm compressive load transfer in cadavers. J Hand Surg [Am] 2005;30:319-25.

11. Stabile KJ, Pfaeffle J, Saris I, Li ZM, Tomaino MM. Structural properties of reconstruction constructs for the interosseous ligament of the forearm. J Hand Surg [Am] 2005;30:312-8.

12. Szabo RM, Hotchkiss RN, Slater RR Jr. The use of frozen-allograft radial head replacement for treatment of established symptomatic proximal translation of the radius: Preliminary experience in five cases. J Hand Surg [Am] 1997;22:269-78. 